\title{
A Phase II Study of Poziotinib in Patients with Epidermal Growth Factor Receptor (EGFR)-Mutant Lung Adenocarcinoma Who Have Acquired Resistance to EGFR-Tyrosine Kinase Inhibitors
}

\author{
Ji-Youn Han, MD, PhD' \\ Ki Hyeong Lee, MD, PhD² \\ Sang-We Kim, MD, $\mathrm{PhD}^{3}$ \\ Young Joo Min, MD, PhD ${ }^{4}$ \\ Eunkyung Cho, MD, $\mathrm{PhD}$ \\ Youngjoo Lee, MD ${ }^{1}$ \\ Soo-Hyun Lee, MD ${ }^{1}$ \\ Hyae Young Kim, MD, PhD' \\ Geon Kook Lee, MD, PhD' \\ Byung Ho Nam, PhD ${ }^{1}$ \\ Hyesun Han, MS 6 \\ Jina Jung, $\mathrm{PhD}^{6}$ \\ Jin Soo Lee, MD, $P h D^{1}$
}

${ }^{1}$ Center for Lung Cancer, National Cancer Center, Goyang, ${ }^{2}$ Department of Internal Medicine, Chungbuk National University Hospital, Cheongju, ${ }^{3}$ Department of Internal Medicine, Asan Medical Center, University of Ulsan College of Medicine, Seoul,

${ }^{4}$ Department of Internal Medicine,

Ulsan University Hospital, Ulsan,

${ }^{5}$ Department of Internal Medicine, Gachon University Gil Medical Center, Incheon, ${ }^{6}$ Clinical Research Team,

Hanmi Pharmaceutical Co., Ltd., Seoul, Korea

\section{Purpose}

We examined the efficacy of poziotinib, a second-generation epidermal growth factor receptor (EGFR)-tyrosine kinase inhibitor (TKI) in patients with lung adenocarcinoma with activating EGFR mutations, who developed acquired resistance (AR) to EGFR-TKIs.

\section{Materials and Methods}

This single-arm phase II study included EGFR-mutant lung adenocarcinoma with AR to erlotinib or gefitinib based on the Jackman criteria. Patients received poziotinib $16 \mathrm{mg}$ orally once daily in a 28-day cycle. The primary endpoint was progression-free survival (PFS). Prestudy tumor biopsies and blood samples were obtained to determine resistance mechanisms.

\section{Results}

Thirty-nine patients were treated. Tumor genotyping was determined in 37 patients; 19 EGFR T790M mutations and two PIK3CA mutations were detected in the prestudy tumors, and seven T790M mutations were detected in the plasma assay. Three (8\%; 95\% confidence interval [Cl], 2 to 21$)$ and 17 (44\%; $95 \% \mathrm{Cl}, 28$ to 60$)$ patients had partial response and stable disease, respectively. The median PFS and overall survival were 2.7 months (95\% Cl, 1.8 to 3.7) and 15.0 months ( $95 \% \mathrm{Cl}, 9.5$ to not estimable), respectively. A longer PFS was observed for patients without T790M or PIK3CA mutations in tumor or plasma compared to those with these mutations ( 5.5 months vs. 1.8 months, $p=0.003$ ). The most frequent grade 3 adverse events were rash (59\%), mucosal inflammation (26\%), and stomatitis (18\%). Most patients required one $(n=15)$ or two $(n=15)$ dose reductions.

\section{Conclusion}

Low activity of poziotinib was detected in patients with EGFR-mutant non-small cell lung cancer who developed AR to gefitinib or erlotinib, potentially because of severe-toxicityimposed dose limitation.
Correspondence: Ji-Youn Han, MD, PhD

Center for Lung Cancer, National Cancer Center, 323 Ilsan-ro, Ilsandong-gu, Goyang 10408, Korea Tel: 82-31-920-1154

Fax: 82-31-920-2799

E-mail: jymama@ncc.re.kr

Received February 8, 2016

Accepted April 17, 2016

Published Online May 3, 2016

\section{Key words}

Epidermal growth factor receptor, Poziotinib, Non-small-cell lung carcinoma, T790M, PIK3CA 


\section{Introduction}

Compared with standard chemotherapy, significant improvement in response rate and progression-free survival (PFS) has been reported for epidermal growth factor receptor (EGFR)-tyrosine kinase inhibitors (TKIs) including gefitinib, erlotinib, and afatinib in patients with EGFR-mutant nonsmall cell lung cancer (NSCLC) [1-3]. EGFR-TKIs are now the treatment of choice for patients with EGFR-mutant NSCLC. However, despite the initial response to EGFR-TKIs, most patients develop resistance and finally relapse. Among several mechanisms responsible for acquired resistance (AR) to EGFR-TKIs, the secondary EGFR T790M point mutation in exon 20 is the most common, occurring in approximately $60 \%$ of patients. Other mechanisms include MET, HER2, or EGFR amplification; histologic transformation to small cell lung cancer [4]; epithelial-to-mesenchymal transition signature [5]; and AXL kinase activation [6]. Coexistence of other mutations including PIK3CA, HER2, or BRAF mutations also contributes to the development of AR to EGFR-TKIs $[7,8]$.

Poziotinib (NOV120101) is an oral, irreversible inhibitor of EGFR, HER2, and HER4. In preclinical studies conducted in cell lines and xenograft models of NSCLC, poziotinib showed more potent activity than gefitinib, erlotinib, and even afatinib in lung cancer models with EGFR mutations including T790M mutation [9]. In a phase I study to examine the safety and maximum tolerated dose (MTD) of continuous daily dosing of poziotinib in genetically unselected patients with advanced solid cancers including NSCLC, $20 \%$ of patients $(4 / 20)$ experienced partial response (PR), with an MTD of $18 \mathrm{mg}$ and an acceptable toxicity profile, supporting further clinical development of poziotinib. The recommended phase II dose was $16 \mathrm{mg} /$ day [10]. This phase II open-label, single-arm study was conducted to examine the anticancer activity and safety of poziotinib in patients with advanced or metastatic lung adenocarcinoma with activating EGFR mutations, who developed AR to EGFR-TKIs based on the Jackman criteria [11].

\section{Materials and Methods}

\section{Patients}

This phase II, open-label, single-arm study enrolled patients aged $\geq 20$ years with histopathologically confirmed stage IIIB or IV lung adenocarcinoma from five institutions in Korea. Eligible patients had at least one measurable lesion or, if not measurable, an evaluable lesion according to the
Response Evaluation Criteria in Solid Tumors (RECIST) ver. 1.1 and documented activating EGFR mutations. Patients had received erlotinib or gefitinib as first-line or subsequentline therapy from which they achieved a best overall response of complete response (CR), PR, or stable disease (SD; at least $\geq 6$ months) and progressed within the last 30 days. Patients had an Eastern Cooperative Oncology Group performance status of $0-2$ with a life expectancy of $\geq 12$ weeks; consented to providing tumor tissue samples; and had a white blood cell count of $\geq 4,000 / \mathrm{mm}^{3}$, platelet count $\geq 100,000 / \mathrm{mm}^{3}$, serum creatinine and total bilirubin level $\leq 1.5$ times the upper limit of normal (ULN), and serum aspartate aminotransferase and alanine aminotransferase level $\leq 2.5$ times the ULN.

The exclusion criteria were as follows: patients with unresolved adverse events (AEs) from erlotinib or gefitinib (Common Terminology Criteria for Adverse Events [CTCAE] grade $\geq 2$ ); resistance to erlotinib or gefitinib secondary to transformation to small-cell lung cancer; major surgery or anticancer therapy other than gefitinib and erlotinib within 4 weeks of the start of the study treatment; untreated symptomatic brain metastasis; interstitial lung disease; active infection; cardiovascular disease or condition including New York Heart Association class III or IV heart failure, uncontrolled hypertension, unstable angina pectoris, or myocardial infarction within 6 months of the study start, uncontrolled cardiac arrhythmia, or other clinically relevant abnormalities; resting left ventricular ejection fraction (LVEF) less than the lower normal limit defined by the institution; any gastrointestinal disease or condition having the prominent symptom of diarrhea; a history of malignancy except for treated nonmelanomatous skin cancer, in situ cervix carcinoma, or ductal in situ breast carcinoma; and patients who were pregnant, lactating, or using inadequate contraception.

\section{Treatment and procedures}

Poziotinib was administered orally, consecutively, and once daily in 28-day cycles with a starting dose of $16 \mathrm{mg}$ until progressive disease (PD) or intolerable AEs. For patients who experienced drug-related AEs, treatment with poziotinib was interrupted until the AEs were resolved to CTCAE grade $\leq 1$ or baseline level. The treatment was resumed at the previous dose or a reduced dose by $4 \mathrm{mg}$, and if a dose reduction below $8 \mathrm{mg}$ consecutively once daily was required, the regimen was changed to intermittent dosing (i.e., 2 weeks of 8-mg poziotinib treatment followed by 1 week off treatment). Further dose reduction or up-titration after the reduction was not allowed. Treatment with poziotinib was discontinued permanently in patients requiring a dose reduction below 8-mg intermittent dosing, had not recovered to AEs of CTCAE grade $\leq 1$ or baseline level within 2 weeks, had pneu- 
monia or LVEF reduction of CTCAE grade $\geq 3$, or had interstitial lung disease or pneumonitis of any grade. Supportive care including anti-diarrhea medication, antibiotics, analgesics, and antiemetics as well as treatments for diseases other than lung adenocarcinoma was allowed, and local palliative radiotherapy to improve symptoms such as bone pain, pulmonary occlusion, and skin lesions was also allowed.

Tumors were assessed by spiral computed tomography or magnetic resonance imaging (except for the chest) at baseline (imaging at the time PD from previous EGFR-TKIs was confirmed and could be used if obtained within 2 weeks prior to baseline), week 4 , week 8 , and every 8 weeks thereafter until PD or the start of new anticancer therapy. After discontinuation of treatment, patients were followed-up every 3 months until death or the start of new anticancer therapy. Scans were reviewed both locally and centrally. Tumor response assessments were based on RECIST ver. 1.1. Tumor tissue samples were mandatory at screening from all patients enrolled (except for those with available tumor tissue collected within 2 weeks of the study start) and optional after PD. Blood samples for genotyping were obtained from patients at screening, cycle 3 , and every two cycles thereafter until the end of treatment. Safety assessments were performed at least every cycle using CTCAE ver. 4.0.

The study was approved by the ethics committee of each study institution and conducted in accordance with the Declaration of Helsinki and Good Clinical Practice Guidelines, and all patients provided written informed consent.

\section{Study outcomes}

The primary endpoint of the study was PFS by independent review. The secondary endpoints included PFS rate at week 16, objective response rate (ORR; $\mathrm{CR}+\mathrm{PR})$, disease control rate (DCR; objective response+SD), overall survival (OS), duration of objective response, and duration of disease control. PFS was calculated from the start of treatment to PD or death, whichever occurred first, or was censored at the last imaging date in the absence of PD or death. OS was calculated from the start of treatment to death or was censored at the last date of contact if the patient was alive. Safety assessments included treatment-related AEs, laboratory tests, vital signs, radiography, electrocardiogram (ECG), and LVEF by a multi-gated acquisition scan or echocardiogram.

\section{Prestudy tumor tissue analysis}

DNA extraction and mutation analysis of prestudy tumor samples were performed in a central laboratory (Theragen Etex, Suwon, Korea). Mutational status was analyzed by Ion Torrent deep-amplicon sequencing using an Ion Torrent AmpliSeq Cancer Hotspot customized lung cancer panel
(Thermo Fisher Scientific Inc., Waltham, MA). Fluorescent in situ hybridization (FISH) of MET (c-MET Probe KBI-10719, Kreatech, LG Amsterdam, Netherlands), EGFR, and HER2 was performed on available tumor tissues using the standard protocol [7,12], and MET expression status was also analyzed using immunohistochemistry (IHC) (clone SP44, Ventana, Tucson, AZ). Tumors with a staining-intensity score of 3 and with more than $50 \%$ positive nuclei were considered to have MET overexpression.

\section{Plasma sample analysis}

Circulating cell-free DNA was extracted from $200 \mu \mathrm{L}$ of plasma samples using the QIAamp MinElute virus spin kit (Qiagen, Hilden, Germany) according to the manufacturer's protocol. The purity and concentration of extracted DNA were determined by spectrophotometry (NanoDrop 2000, Thermo Fisher Scientific Inc.). DNA samples with absorption ratios of 260/280 $\mathrm{nm}$ greater than 1.8 were used for subsequent analyses. For plasma samples, only T790M mutation was analyzed using the PANAMutyper R EGFR kit (Panagene, Daejeon, Korea), a new highly sensitive mutation detection kit that uses a peptide nucleic acid clampingassisted fluorescence melting curve analysis method in mutation detection and genotyping.

\section{Statistical analysis}

The sample size $(n=40)$ was selected to provide at least 34 patients with PFS event considering 15\% of follow-up loss with the following assumption: in a 12-month period of patient enrollment, a total of 34 PFS events in the study would have a $90 \%$ power for detection of a 1.8-month improvement of median PFS with poziotinib versus historical control where the conventional anticancer chemotherapies had been implemented (median PFS, 4.5 months vs. 2.7 months for poziotinib and historical control $[13,14]$, respectively), with a one-sided significance level of $5 \%$. The primary endpoint, median PFS, and the secondary endpoints including median OS, time to progression, time to objective response, duration of objective response, and duration of disease control were calculated from Kaplan-Meier estimates with $95 \%$ confidence interval (CI). For other secondary endpoints including PFS rate at 16 weeks, ORR, and DCR, the number and proportion of patients was calculated with $95 \%$ CIs. Statistical analysis for subgroups based on mutational status was performed using log-rank, Fisher exact, or Pearson's chi-square tests depending on the types of variables. Efficacy assessments were performed in the full analysis set consisting of patients who received at least one dose of poziotinib with at least one evaluation of the primary efficacy endpoint after baseline. Safety data was summarized 
Table 1. Patient demographics and baseline characteristics

\begin{tabular}{lc}
\hline Characteristic & No. $(\%)$ \\
Age, median (range, yr) & $62(43-84)$ \\
Sex & \\
$\quad$ Male & $10(26)$ \\
Female & $29(74)$ \\
\hline Smoking status & \\
\hline Never & $30(77)$ \\
\hline Former & $9(23)$ \\
Current & 0 \\
\hline Smoking pack year, median (range) & $22(17-28)$ \\
\hline ECOG PS & $6(15)$ \\
\hline 0 & $30(77)$ \\
\hline 1 & $3(78)$ \\
\hline 2 & \\
\hline Therapy line of previous EGFR-TKI & $27(69)$ \\
\hline First & $11(28)$ \\
\hline Second & 0 \\
\hline Third & $1(3)$ \\
\hline Fourth & $13.1(3.4-33.2)$ \\
\hline Duration of previous EGFR-TKI, & \\
median (range, mo) & $36(92)$ \\
Best response to previous EGFR-TKI & \\
\hline Complete response & \\
\hline Partial response & \\
\hline Stable disease & \\
\hline
\end{tabular}

ECOG PS, Eastern Cooperative Oncology Group performance status; EGFR, epidermal growth factor receptor; TKI, tyrosine kinase inhibitor.

descriptively in patients who received at least one dose of poziotinib.

\section{Results}

\section{Patient characteristics and disposition}

Forty patients were enrolled between December 2012 and September 2013. Thirty-nine patients received at least one dose of poziotinib with at least one tumor assessment after baseline and were included in the full analysis set and safety set. One patient who did not receive the study drug was withdrawn from the study and excluded from the data analysis. Demographics and baseline characteristics of patients are shown in Table 1 . The median age was 62 years (range, 43 to 84 years), and the majority of patients were
Table 2. Response to treatment with poziotinib by independent review

\begin{tabular}{|lc}
\hline Variable & Total (n=39) \\
\hline PFS events, $\mathbf{n}(\%)$ & $34(87)$ \\
\hline Estimated PFS, median (95\% CI, mo) & $2.7(1.8-3.7)$ \\
\hline PFS events at week 16, $\mathbf{n}(\%)$ & $24(62)$ \\
\hline Estimated PFS at week $\mathbf{1 6}$ (95\% CI, \%) & $35(20-51)$ \\
\hline Deaths, $\mathbf{n}(\%)$ & $20(51)$ \\
\hline Estimated OS, median (95\% CI, mo) & $15.0(9.5-\mathrm{NE})$ \\
\hline Best response, $\mathbf{n} \mathbf{( \% )}$ & \\
\hline CR & 0 \\
\hline PR & $3(8)$ \\
\hline SD & $17(44)$ \\
\hline PD & $18(46)$ \\
\hline Not evaluable & $1(3)$ \\
\hline ORR (95\% CI, \%) & $8(2-21)$ \\
\hline DCR (95\% CI, \%) & $51(35-68)$ \\
\hline Estimated duration of response, median & $4.5(3.7-4.6)$ \\
\hline (95\% CI, mo) & \\
\hline Estimated duration of disease control, & $3.7(1.8-3.8)$ \\
median (95\% CI, mo) & \\
\hline
\end{tabular}

PFS, progression-free survival; $\mathrm{CI}$, confidence interval; OS, overall survival; $\mathrm{NE}$, not estimable; $\mathrm{CR}$, complete response; $\mathrm{PR}$, partial response; $\mathrm{SD}$, stable disease; $\mathrm{PD}$, progressive disease; ORR, objective response rate; $\mathrm{DCR}$, disease control rate.

women $(74 \%)$ and never-smokers $(77 \%)$. All patients had stage IV disease. Most patients received gefitinib or erlotinib as first-line $(69 \%)$ or second-line $(28 \%)$ therapy with a median treatment duration of 10.4 months (range, 3.4 to 33.2 months). The best response to previous EGFR-TKIs was predominantly PR (92\%). At the time of data cut-off (September $1,2014)$, all patients in the full analysis set, except for one who achieved PR with poziotinib, discontinued treatment for the following reasons: lack of efficacy or PD $(n=28)$, voluntary withdrawal of consent $(n=7)$, AEs $(n=2)$, or other reason $(n=1)$.

\section{Efficacy assessments}

A summary of the efficacy measures for poziotinib by independent review is shown in Table 2. The median PFS at the time of analysis (January 7, 2015) was 2.7 months (95\% CI, 1.8 to 3.7), and the PFS rate was $35 \%$ at week $16(95 \% \mathrm{CI}$, 20 to 51). The best response changes from baseline in tumor lesions were evaluated in 35 patients with measurable lesions at baseline (Fig. 1). Three patients achieved PR (ORR, 8\%; 95\% CI, 2 to 21). The Kaplan-Meier estimate of median duration of objective response was 4.5 months (95\% CI, 3.7 to 4.6$)$, and the DCR was $51 \%$ (95\% CI, 35 to 68$)$. At the data 
cut-off, 20 OS events were reported. The Kaplan-Meier estimate of median OS was 15.0 months $(95 \%$ CI, 9.5 to not estimable).

\section{The possible mechanism of AR to EGFR-TKIs}

Of 39 patients who received poziotinib, mutational status in prestudy tumor tissues was determined in 37 patients (Fig. 2A), but not in two patients because of inadequate tissue samples. All tumor samples were reviewed by a thoracic pathologist (G.K.L.). Among the 37 patients, 19 (51\%) had T790M mutation and two (5\%) had PIK3CA mutations, and one patient had EGFR wild type.

Four patients donated tumor tissue at the end of treatment, and all were T790M-positive at screening and at the end of treatment.

To further elucidate the mechanisms of AR, plasma EGFR T790M genotyping was performed in all patients $(n=39)$, which identified seven additional cases of T790M mutation, which were not detected in tumor tissue samples. The performance of plasma T790M genotyping is summarized in Supplementary Table 1.

Sufficient tumor tissue was available from 35 patients for $M E T, E G F R$, and HER2 FISH analysis. Among three patients with EGFR amplifications, two overlapped with EGFR
T790M mutation. No MET or HER2 amplification was detected. Of 35 patients tested using IHC, five patients had MET overexpression and four overlapped with EGFR T790M mutation. The frequencies of possible AR mechanisms are shown in Fig. 2B. Overall, of 39 patients, 26 patients (67\%) developed AR to prior EGFR-TKIs due to acquisition of T790M mutation.

\section{Clinical outcome according to mutational status}

In subgroup analysis on the EGFR T790M mutational status in the prestudy tumor tissue, the estimated median PFS in patients with EGFR T790M mutation (2.7 months; $95 \%$ CI, 1.7 to 3.9) was shorter than that for patients without T790M mutation (3.7 months; 95\% CI, 1.4 to 5.4; $\mathrm{p}=0.329$ ) (Supplementary Fig. S1A). Similarly, patients with the EGFR T790M or PIK3CA mutations in prestudy tumor tissue had a shorter median PFS (1.9 months; 95\% CI, 0.9 to 3.6) than those without these mutations (3.7 months; 95\% CI, 1.7 to 5.5; $\mathrm{p}=0.103$ ) (Supplementary Fig. S1B). However, these differences were not statistically significant.

Regarding the EGFR T790M mutation in baseline plasma samples, a significantly shorter median PFS (1.8 months; 95\% CI, 1.7 to 1.9) was observed for patients with EGFR T790M mutations than for those without these mutations (3.7

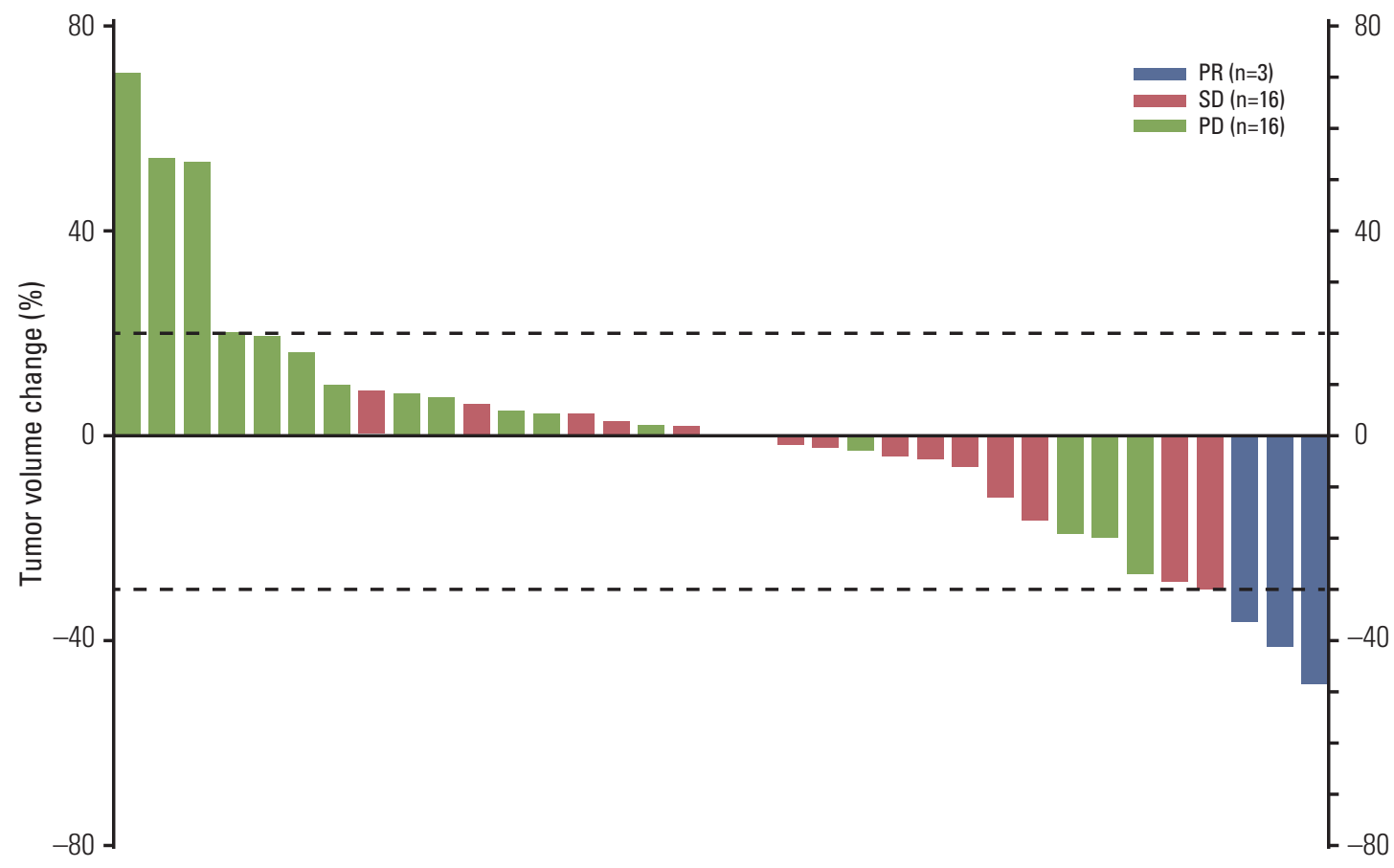

Fig. 1. Best response changes from baseline in tumor lesions: blue (partial response, PR), red (stable disease, SD), and green (progressive disease, $\mathrm{PD}$ ). 

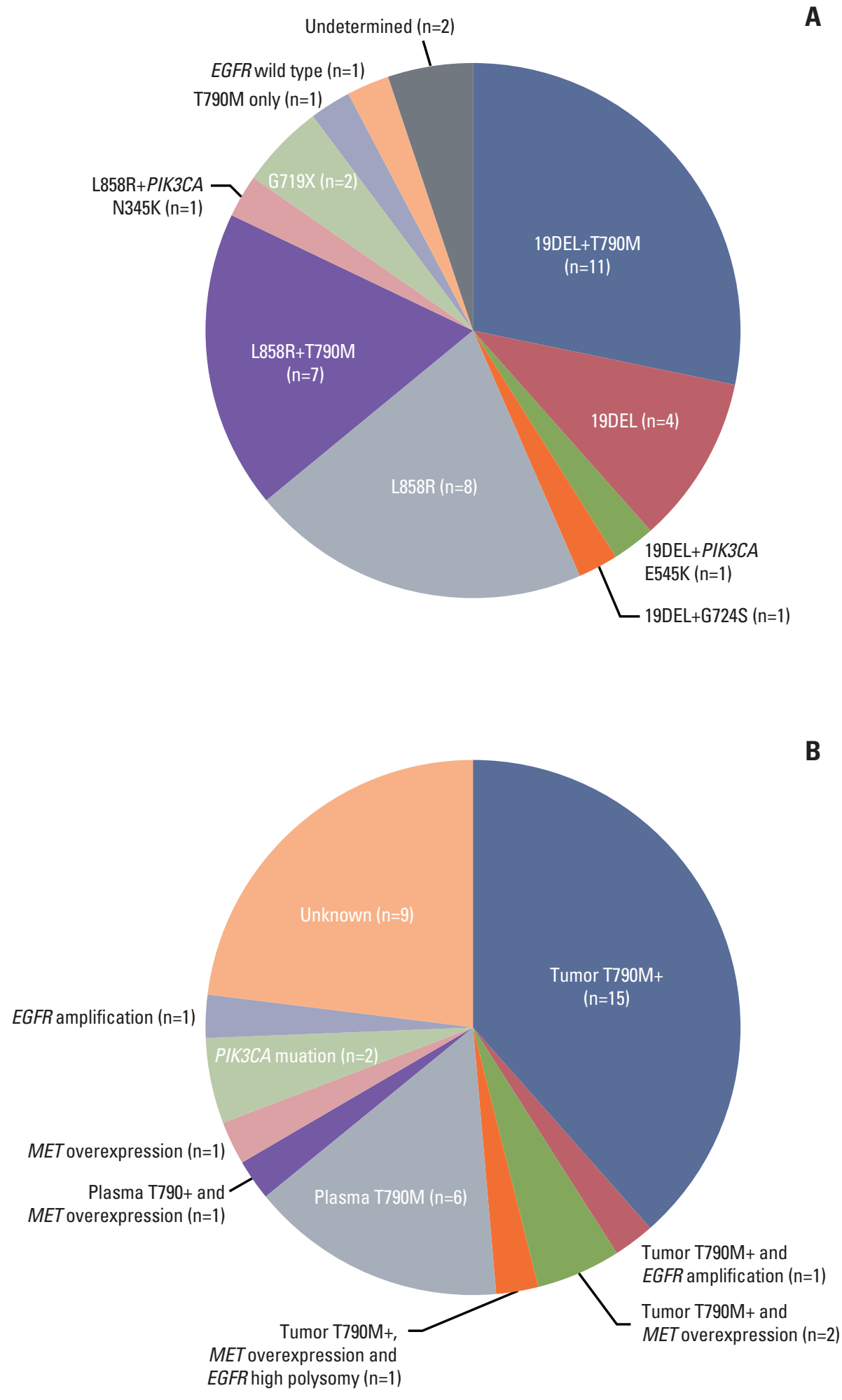

Fig. 2. (A) Mutational status in the prestudy tumor tissues. (B) Possible mechanism of acquired resistance to prior epidermal growth factor receptor (EGFR)-tyrosine kinase inhibitors. 
Table 3. TEAEs by CTCAE grade reported in $\geq 5 \%$ of patients

\begin{tabular}{|c|c|c|c|c|}
\hline Preferred term & Any grade & Grade 1 & Grade 2 & Grade 3 \\
\hline No. of patients & $39(100)$ & $39(100)$ & $39(100)$ & $39(100)$ \\
\hline Total with adverse events & $39(100)$ & $39(100)$ & $37(95)$ & 37 (95) \\
\hline Diarrhoea & $36(92)$ & $30(77)$ & $17(44)$ & $4(10)$ \\
\hline Rash & $30(77)$ & $14(36)$ & $17(44)$ & $23(59)$ \\
\hline Pruritus & $25(64)$ & $10(26)$ & $17(44)$ & $2(5)$ \\
\hline Stomatitis & $23(59)$ & $11(28)$ & $13(33)$ & $7(18)$ \\
\hline Paronychia & $21(54)$ & $8(21)$ & $13(33)$ & $2(5)$ \\
\hline Decreased appetite & $19(49)$ & $12(31)$ & $8(21)$ & $5(13)$ \\
\hline Mucosal inflammation & $18(46)$ & $8(21)$ & $9(23)$ & $10(26)$ \\
\hline Dry skin & $15(38)$ & $8(21)$ & $7(18)$ & $1(3)$ \\
\hline Fatigue & $12(31)$ & $7(18)$ & $7(18)$ & $1(3)$ \\
\hline Dyspepsia & $8(21)$ & $6(15)$ & $2(5)$ & 0 \\
\hline Hypokalaemia & $7(18)$ & $2(5)$ & $2(5)$ & $4(10)$ \\
\hline Alopecia & $6(15)$ & $6(15)$ & 0 & 0 \\
\hline Dermatitis acneiform & $5(13)$ & $2(5)$ & 0 & $4(10)$ \\
\hline Weight decreased & $5(13)$ & $3(8)$ & $2(5)$ & $1(3)$ \\
\hline Skin exfoliation & $4(10)$ & $3(8)$ & $1(3)$ & 0 \\
\hline Palmar-plantar erythrodysaesthesia syndrome & $3(8)$ & $1(3)$ & $2(5)$ & $1(3)$ \\
\hline Dysphagia & $3(8)$ & $1(3)$ & $2(5)$ & 0 \\
\hline Nausea & $3(8)$ & $2(5)$ & $1(3)$ & 0 \\
\hline Pneumothorax & $3(8)$ & $3(8)$ & 0 & 0 \\
\hline Muscular weakness & $3(8)$ & 0 & $3(8)$ & 0 \\
\hline Hirsutism & $2(5)$ & $2(5)$ & 0 & 0 \\
\hline Pain of skin & $2(5)$ & 0 & $2(5)$ & 0 \\
\hline Petechiae & $2(5)$ & $1(3)$ & $1(3)$ & 0 \\
\hline Vomiting & $2(5)$ & $1(3)$ & 0 & $1(3)$ \\
\hline Abdominal pain upper & $2(5)$ & 0 & $2(5)$ & 0 \\
\hline Asthenia & $2(5)$ & 0 & $2(5)$ & 0 \\
\hline Conjunctivitis & $2(5)$ & 0 & $2(5)$ & $1(3)$ \\
\hline Nail infection & $2(5)$ & 0 & $2(5)$ & 0 \\
\hline Blood creatinine increased & $2(5)$ & $1(3)$ & $1(3)$ & 0 \\
\hline Rhinorrhoea & $2(5)$ & $1(3)$ & $1(3)$ & 0 \\
\hline Eye discharge & $2(5)$ & $2(5)$ & $1(3)$ & 0 \\
\hline Dysuria & $2(5)$ & $1(3)$ & $1(3)$ & 0 \\
\hline Anaemia & $2(5)$ & 0 & $1(3)$ & $1(3)$ \\
\hline
\end{tabular}

Values are presented as number (\%). No grade 4 or 5 events were reported. TEAE, treatment-emergent adverse event; CTCAE, Common Terminology Criteria for Adverse Events.

months; 95\% CI, 3.5 to 3.8; $\mathrm{p}=0.006$ ) (Supplementary Fig. S1C). When combining the mutation results in tumor and plasma, a more prominent difference in PFS was observed. Patients with T790M or PIK3CA mutation (1.8 months; $95 \%$ CI, 1.7 to 1.9 ) had significantly shorter estimated median PFS than those without these mutations (5.5 months; $95 \%$ CI, 3.1 to 7.8; $\mathrm{p}=0.003$ ) (Supplementary Fig. S1D).

\section{Safety and tolerability}

Patients remained on poziotinib for a median of 58 days (range, 17 to 443 days); $23 \%$ of patients (9/39) continued poziotinib without dose reduction throughout the treatment and others $(77 \%)$ required at least one dose reduction; 15 $(38 \%)$ required one dose reduction, and $15(38 \%)$ required two dose reductions.

All patients treated with poziotinib experienced at least one treatment-related $\mathrm{AE}$; the most frequently reported $\mathrm{AEs}$ 
( $\geq 30 \%$ of patients) included diarrhea, rash, pruritus, stomatitis, paronychia, decreased appetite, mucosal inflammation, dry skin, and fatigue (Table 3). The most frequently reported grade 3 AEs (reported in 95\% of patients) were rash, mucosal inflammation, stomatitis, decreased appetite, dermatitis acneiform, diarrhea, and hypokalemia. Overall, 17 serious adverse events were reported in eight patients $(21 \%)$, with diarrhea being the most frequent $(4 / 39 ; 10 \%)$ followed by stomatitis and rash $(2 / 39 ; 5 \%$, each). Rash was the most common reason for dose reduction $(17 / 39 ; 44 \%)$ and dose interruption $(9 / 39 ; 23 \%)$. Two events (one myositis and one rash) resulted in permanent discontinuation and there was no treatment-related death. Poziotinib was not considered to be related to any significant changes in laboratory tests, vital signs, or other safety endpoints (including chest radiography, ECG, and LVEF).

\section{Discussion}

This study assessed the efficacy and safety of poziotinib, a pan-EGFR-TKI, in patients with EGFR-mutant NSCLC who developed AR to gefitinib or erlotinib. Patients enrolled in this trial were required to meet the eligibility criteria based on Jackman's clinical definition of AR to EGFR-TKIs in NSCLC [10]. Similar to other reports, T790M mutation was the most common cause of AR. Other mechanisms included PIK3CA mutation, EGFR amplification, or MET overexpression. In this heterogeneous patient population, overall ORR was $8 \%$ and DCR was $51 \%$. The Kaplan-Meier estimate of median PFS (2.7 months) did not reach the extent of the original assumption used for calculation of the sample size (4.5 months). However, patients without T790M mutation had better median PFS than those with T790M mutation. These results suggest that poziotinib may not overcome AR secondary to T790M mutation. In addition, when combining the results of patients with or without T790M or PIK3CA mutations in tissue or plasma, patients without these mutations had significantly longer PFS than those with these mutations (5.5 months vs. 1.8 months, $\mathrm{p}=0.003$ ), which exceeds the primary endpoint results of this study. This finding suggests that poziotinib may not overcome AR due to EGFR T790M or PIK3CA mutation. However, it can be considered as a new treatment option for patients who develop AR not due to these mutations.

As an irreversible EGFR-TKI, poziotinib has shown in vitro activity for EGFR T790M mutant NSCLC cells. Several irreversible EGFR-TKIs including afatinib, dacomitinib, and neratinib have been evaluated in patients previously treated with gefitinib or erlotinib, yielding a response rate of less than $10 \%$ and PFS of less than 4 months [15-17]. Despite their preclinical activity against T790M mutation, these drugs failed to demonstrate adequate efficacy in these populations. In addition, their potent wild type EGFR-inhibiting activity leads to intolerable skin and gastrointestinal toxicities, and poziotinib was associated with serious skin rash and diarrhea. Finally, most patients required frequent dose reduction, which may further decrease the potential for inhibiting T790M mutation.

Recent progress has been made in the treatment of EGFR T790M mutant NSCLC with mutant-selective EGFR-TKIs including AZD9291, rociletinib, and HM61713. Response rates of over $50 \%$ have been reported in patients with T790M mutation. In addition, compared with other EGFR-TKIs developed so far, these drugs have more favorable toxicity profiles [18-20]. Thus, these mutant-selective EGFR-TKIs would be a promising treatment option for patients with EGFR T790M mutant NSCLC.

Molecular analysis at the time of progression on EGFR-TKI therapy is important in deciding on the next treatment plan. Tumor tissue is the preferred sample type when available; however, there is increasing evidence that plasma can be used as a substitute for molecular analysis in NSCLC [21]. In this study, we also examined the utility of plasma samples for EGFR T790M mutation analysis. EGFR T790M mutation rates were higher in tumor tissue $(19 / 37,51 \%$ in evaluable samples) than in plasma $(18 / 39,46 \%)$. The concordance was $62 \%$, with a specificity of $67 \%$ and sensitivity of $58 \%$ (Supplementary Table 1). Despite the low concordance rate observed here, the plasma T790M results were more predictive for efficacy compared with tissue results (Supplementary Fig. S1C), and five out of six patients with T790Mpositive plasma but T790M-negative tissue showed PD with poziotinib. Thus, further clinical evaluation of the plasma assay is required.

\section{Conclusion}

In this explorative phase II study poziotinib provided modest clinical benefit in patients with advanced or metastatic lung adenocarcinoma having progressed on erlotinib or gefitinib. This study provided no obvious clinical evidence showing that poziotinib may overcome AR secondary to EGFR T790M mutation. 


\section{Electronic Supplementary Material}

Supplementary materials are available at Cancer Research and Treatment website (http:// www.e-crt.org).

\section{Acknowledgments}

This study was supported by National OncoVenture and Hanmi Pharmaceutical Co., Ltd. (NCT01718847).

\section{Conflicts of Interest}

Hyesun Han and Jina Jung are fulltime employees of the Hanmi Pharmaceutical Co., Ltd., Seoul, South Korea.

\section{References}

1. Maemondo M, Inoue A, Kobayashi K, Sugawara S, Oizumi S, Isobe $\mathrm{H}$, et al. Gefitinib or chemotherapy for non-small-cell lung cancer with mutated EGFR. N Engl J Med. 2010;362:23808.

2. Zhou C, Wu YL, Chen G, Feng J, Liu XQ, Wang C, et al. Erlotinib versus chemotherapy as first-line treatment for patients with advanced EGFR mutation-positive non-smallcell lung cancer (OPTIMAL, CTONG-0802): a multicentre, open-label, randomised, phase 3 study. Lancet Oncol. 2011;12: 735-42.

3. Yang JC, Shih JY, Su WC, Hsia TC, Tsai CM, Ou SH, et al. Afatinib for patients with lung adenocarcinoma and epidermal growth factor receptor mutations (LUX-Lung 2): a phase 2 trial. Lancet Oncol. 2012;13:539-48.

4. Sequist LV, Waltman BA, Dias-Santagata D, Digumarthy S, Turke AB, Fidias $\mathrm{P}$, et al. Genotypic and histological evolution of lung cancers acquiring resistance to EGFR inhibitors. Sci Transl Med. 2011;3:75ra26.

5. Suda K, Tomizawa K, Fujii M, Murakami H, Osada H, Maehara $Y$, et al. Epithelial to mesenchymal transition in an epidermal growth factor receptor-mutant lung cancer cell line with acquired resistance to erlotinib. J Thorac Oncol. 2011;6: 1152-61.

6. Zhang Z, Lee JC, Lin L, Olivas V, Au V, LaFramboise T, et al. Activation of the AXL kinase causes resistance to EGFR-targeted therapy in lung cancer. Nat Genet. 2012;44:852-60.

7. Yu HA, Arcila ME, Rekhtman N, Sima CS, Zakowski MF, Pao $\mathrm{W}$, et al. Analysis of tumor specimens at the time of acquired resistance to EGFR-TKI therapy in 155 patients with EGFRmutant lung cancers. Clin Cancer Res. 2013;19:2240-7.

8. Ohashi K, Sequist LV, Arcila ME, Moran T, Chmielecki J, Lin $\mathrm{YL}$, et al. Lung cancers with acquired resistance to EGFR inhibitors occasionally harbor BRAF gene mutations but lack mutations in KRAS, NRAS, or MEK1. Proc Natl Acad Sci U S A. 2012;109:E2127-33.

9. Cha MY, Lee KO, Kim M, Song JY, Lee KH, Park J, et al. Anti- tumor activity of HM781-36B, a highly effective pan-HER inhibitor in erlotinib-resistant NSCLC and other EGFR-dependent cancer models. Int J Cancer. 2012;130:2445-54.

10. Kim YJ, Oh J, Kim TM, Han SW, Lee KW, Kim JH, et al. Phase I study to evaluate the safety and to assess the food effect of HM781-36B, a novel pan-HER inhibitor continuously given in patients with advanced solid tumors. J Clin Oncol. 2013;31 (Suppl):Abstr 2565.

11. Jackman D, Pao W, Riely GJ, Engelman JA, Kris MG, Janne PA, et al. Clinical definition of acquired resistance to epidermal growth factor receptor tyrosine kinase inhibitors in nonsmall-cell lung cancer. J Clin Oncol. 2010;28:357-60.

12. Sequist LV, Martins RG, Spigel D, Grunberg SM, Spira A, Janne PA, et al. First-line gefitinib in patients with advanced non-small-cell lung cancer harboring somatic EGFR mutations. J Clin Oncol. 2008;26:2442-9.

13. Kim ES, Hirsh V, Mok T, Socinski MA, Gervais R, Wu YL, et al. Gefitinib versus docetaxel in previously treated non-smallcell lung cancer (INTEREST): a randomised phase III trial. Lancet. 2008;372:1809-18.

14. Gridelli C, Ciardiello F, Gallo C, Feld R, Butts C, Gebbia V, et al. First-line erlotinib followed by second-line cisplatin-gemcitabine chemotherapy in advanced non-small-cell lung cancer: the TORCH randomized trial. J Clin Oncol. 2012;30:3002-11.

15. Sequist LV, Besse B, Lynch TJ, Miller VA, Wong KK, Gitlitz B, et al. Neratinib, an irreversible pan-ErbB receptor tyrosine kinase inhibitor: results of a phase II trial in patients with advanced non-small-cell lung cancer. J Clin Oncol. 2010;28: 3076-83.

16. Miller VA, Hirsh V, Cadranel J, Chen YM, Park K, Kim SW, et al. Afatinib versus placebo for patients with advanced, metastatic non-small-cell lung cancer after failure of erlotinib, gefitinib, or both, and one or two lines of chemotherapy (LUXLung 1): a phase $2 b / 3$ randomised trial. Lancet Oncol. 2012; 13:528-38.

17. Ellis PM, Shepherd FA, Millward M, Perrone F, Seymour L, 
Liu G, et al. Dacomitinib compared with placebo in pretreated patients with advanced or metastatic non-small-cell lung cancer (NCIC CTG BR.26): a double-blind, randomised, phase 3 trial. Lancet Oncol. 2014;15:1379-88.

18. Janne PA, Yang JC, Kim DW, Planchard D, Ohe Y, Ramalingam SS, et al. AZD9291 in EGFR inhibitor-resistant nonsmall-cell lung cancer. N Engl J Med. 2015;372:1689-99.

19. Sequist LV, Soria JC, Goldman JW, Wakelee HA, Gadgeel SM, Varga A, et al. Rociletinib in EGFR-mutated non-small-cell lung cancer. N Engl J Med. 2015;372:1700-9.

20. Tan CS, Cho BC, Soo RA. Next-generation epidermal growth factor receptor tyrosine kinase inhibitors in epidermal growth factor receptor-mutant non-small cell lung cancer. Lung Cancer. 2016;93:59-68.

21. Douillard JY, Ostoros G, Cobo M, Ciuleanu T, McCormack R, Webster A, et al. First-line gefitinib in Caucasian EGFR mutation-positive NSCLC patients: a phase-IV, open-label, singlearm study. Br J Cancer. 2014;110:55-62. 